\title{
Audiencia y programación en Televisión Española: del ocaso del modelo paleotelevisivo al umbral del neotelevisivo
}

\author{
Marta RoEL \\ Universidad de Murcia \\ mroel@um.es
}

Recibido: 04/02/2014

Aceptado: 10/03/2014

\begin{abstract}
Resumen
Partiendo de las elecciones generales de finales de 1982 que dan la victoria al PSOE de Felipe González, en este artículo se realiza una aproximación al estudio cuantitativo de la audiencia de TVE en la etapa final del monopolio de la televisión pública en España, estableciendo posibles vínculos entre el comportamiento de las audiencias y los modelos de programación ofertados por TVE1 y TVE2.

Palabras clave: TVE, audiencia, programación, democracia, España.
\end{abstract}

\section{Audience and programming in Televisión Española: from the paleotelevi- sion's decline to the neotelevision model's threshold}

\begin{abstract}
Starting from the democratic elections in late 1982 to give victory to the Felipe González's socialist government, in this paper an approach is made to the quantitative study of the audience of TVE in the final stage of the monopoly of public television in Spain, establishing possible links between the conduct of audience and programming models offered by TVE1 and TVE2.
\end{abstract}

Keywords: TVE, audience, programming, democracy, Spain.

Referencia normalizada: ROEL, Marta (2014): “Audiencia y programación en Televisión Española: del ocaso del modelo paleotelevisivo al umbral del neotelevisivo". Estudios sobre el Mensaje Periodístico, Vol. 20, Núm. especial, pp. 157-175. Madrid, Servicio de Publicaciones de la Universidad Complutense.

\section{Sumario}

1. Introducción. 2. Metodología de investigación. 3. El Estudio General de Medios: método de medición de audiencias en constante evolución. 4. Evolución de la audiencia en televisión (1983-1990): de las audiencias cautivas a las audiencias compartidas. 5 . Audiencia y programación en el comienzo del proceso de democratización de Televisión Española: el ocaso del modelo paleotelevisivo; 5.1. Estructura de la audiencia en TVE; 5.2. El modelo de programación generalista en TVE; 5.3. Análisis del comportamiento de las audiencias. 6. Audiencia y programación en el umbral de la Neotelevisión; 6.1. Estructura de la audiencia en TVE; 6.2. Modelos de programación cada vez más diferenciados; 6.3. Análisis del comportamiento de las audiencias de TVE en el comienzo de la desregulación. 7. Conclusiones. 8. Referencias. 9. Anexos.

\section{Introducción}

En octubre de 1982 se celebran en España elecciones generales. A partir de entonces será el PSOE a través de sucesivos gobiernos presididos durante 14 años por Felipe González Márquez, el encargado de llevar las riendas del país y de intentar consolidar la democracia en España -tarea ya iniciada años antes por los gobiernos de UCDregenerando el conjunto de instituciones del Estado pero también la gestión de la televisión pública estatal, objeto principal del presente artículo. 
Recordemos que el monopolio de la televisión pública en España se extiende hasta 1990, fecha en que comienzan las emisiones regulares de las televisiones privadas (gestión indirecta del servicio público de televisión). Este momento histórico nos sirve asimismo para cerrar nuestro período de estudio porque esa ruptura del monopolio de la televisión pública trae consigo el inicio de una nueva etapa, la desregulación del sector, y de un nuevo modelo de programación, que avanza -en palabras de Umberto Eco (1986)- de la Paleotelevisión ${ }^{1}$ a la Neotelevisión ${ }^{2}$ (Cortés, 1999). En esta nueva etapa de coexistencia del modelo público y comercial, ambos modelos lejos de diferenciarse -como cabría esperar- compiten abiertamente por los mismos públicos con estrategias de programación y contenidos similares (Roel, 2006). Y ése será el procedimiento de actuación prácticamente hasta la actualidad.

El proceso de democratización de Televisión Española se había iniciado en 1980 con la aprobación del Estatuto de la Radio y la Televisión-ERT (Ley 4/1980 de 10 de enero. BOE $n^{\circ} 11$ de 12 de enero) que diseña el esperado y necesario nuevo marco jurídico para RTVE, desarrollando el artículo 20.3 de la Constitución española de 1978 (Fernández y Santana 2000). Para facilitar la gestión, este estatuto crea tres sociedades estatales (RNE, RCE y TVE) pertenecientes al Ente público RTVE y traza un nuevo modelo organizativo cuya gestión a partir de entonces, recaerá sobre el Presidente del ente público, nombrado directamente por el gobierno, el Consejo de Administración, 12 miembros nombrados por el Congreso de los Diputados y el Senado y los Consejos Asesores, un órgano meramente consultivo.

Este sistema organizativo deja entrever desde sus inicios, las continuas injerencias políticas derivadas precisamente de la estructura diseñada, uno de los males que ha acompañado a RTVE a lo largo de su existencia hasta la actualidad ${ }^{3}$.

1 La Paleotelevisión es la etapa de monopolio absoluto de la televisión pública que en España abarca desde el inicio de las emisiones regulares el 28 de octubre de 1956 hasta la aparición de la televisión privada en torno a 1990. Si bien a partir de 1983 la televisión de cobertura estatal comienza a compartir el monopolio televisivo con las nacientes televisiones autonómicas públicas.

La Paleotelevisión se caracteriza, por tanto, por la ausencia de competitividad y de libre mercado y por impulsar un modelo de programación generalista de servicio público y de corte familiar, basado en los principios tradicionales de informar, educar y entretener a un público heterogéneo y amplio.

2 La Neotelevisión se inicia con la ruptura del monopolio de la televisión pública y el comienzo de una etapa de desreglamentación que posibilita la aparición de las primeras televisiones privadas y el desarrollo del sector televisivo en un entorno más competitivo, hecho que en España sucede -tal y como se ha indicado anteriormente- a partir de 1990. Desde entonces, la televisión pública debe aprender a familiarizarse con la nueva oferta comercial y asumir las nuevas reglas del juego. Estamos ante una nueva edad televisiva en la que las televisiones públicas y privadas operan en abierta competencia en una batalla comercial en la que sus programaciones se disputan minuto a minuto los mayores índices de audiencia. Esta competitividad que transforma la programación de las cadenas de televisión existentes y estructura la de las recientes, sienta las bases de la Neotelevisión.

3 Si bien, desde 2006 existe un nuevo marco normativo regulador para RTVE que teóricamente pretendía erradicar estas actuaciones, las acusaciones o sospechas de intromisiones políticas siguen vigentes a lo largo de los años tal y como recogen algunos medios de comunicación: http://sociedad.elpais.com/sociedad/2013/01/29/actualidad/1359491498_883641.html [fecha de consulta, 12 de julio de 2013]. 
El ERT plantea asimismo una novedad, la posibilidad de atribuir la gestión directa de un canal de televisión a otros entes públicos, las comunidades autónomas. Posteriormente llegará algo determinante en la conformación de una nueva Era televisiva: se reconoce que la televisión podría ser susceptible de gestión directa o indirecta (Bustamante, 2002), lo cual se materializará en la aprobación de la Ley de televisión privada (BOE n ${ }^{\circ} 108$ de 5 de mayo) y en las primeras concesiones en agosto de 1989.

Por tanto, en estas afirmaciones está latente el germen estructural de lo que con el paso de los años, en torno a 1990 (momento en el que concluimos nuestro estudio), será el dibujo de la estructura de la televisión en España constituida por la televisión pública estatal (TVE1 y TVE2), las televisiones públicas autonómicas ${ }^{4}$ (ETB1, ETB2, TV3, Canal 33, TVG, Canal Sur, Telemadrid y Canal 9) y las tres primeras televisiones privadas (Antena 3, Telecinco y Canal Plus).

\section{Metodología de investigación}

Partiendo de este contexto temporal, político y organizativo, en este artículo se pretende realizar una aproximación, fundamentalmente cuantitativa, a la audiencia de Televisión Española y al modelo de programación predominante en la etapa final de monopolio de las televisiones públicas, sabiendo que es precisamente durante estos años cuando la cadena estatal comienza a compartir sus audiencias, primero con las nacientes televisiones autonómicas y, posteriormente con las privadas.

Para abordar esta investigación, se ha acudido básicamente a los datos de audiencia del Estudio General de Medios (EGM) que utiliza un sistema de medición de audiencias cuantitativo, predominante durante aquel periodo de tiempo, a través de la realización de entrevistas, hasta que hacia 1988 tienen lugar las experiencias iniciales de audimetría en España, método de medición de audiencias de televisión hegemónico desde comienzos de la década de los noventa del siglo XX hasta la actualidad.

Asimismo, se ha accedido a las parrillas de programación de Televisión Española y a información hemerográfica con objeto de poder establecer posibles vínculos entre las audiencias (sus perfiles y horarios preferidos) y los contenidos ofertados por los dos canales de TVE.

Con objeto de analizar los perfiles de audiencia y los datos cuantitativos por franjas horarias, hemos seleccionado los datos facilitados por la $\mathrm{AIMC}^{6}$-EGM que hacen referencia a dos periodos temporales coincidentes con el comienzo y el final de nuestro estudio (noviembre de 1982 a octubre de 1983 y febrero a noviembre de 1990).

Uno de los principales inconvenientes o limitaciones que nos hemos encontrado en el procesado de estos datos y su adaptación a nuestro objeto de estudio, ha sido la evolución del diseño muestral del EGM que a lo largo de los años se ha ido modificando, tal y como se explica en el siguiente epígrafe. Esta adaptación a nuevas necesidades afecta entre otras variables, a la diversidad del cómputo de población estudiada

4 Sobre el origen de las televisiones autonómicas en España puede consultarse Guimerá y Blasco, 2012.

Véase Roel, 2006.

6 Asociación para la Investigación de los Medios de Comunicación. 
(desde 1988 incorpora, por ejemplo, la población de núcleos rurales y la de 14 años), al diseño de las franjas de edad y a los periodos horarios.

Ello explica, en parte el diseño estructural del presente artículo ya que si bien hemos seleccionado los datos iniciales y finales del período de estudio, tal y como se ha explicado, hemos optado por no establecer comparativas entre los datos cuantitativos de ambos periodos, tan solo para dar cuenta de la evolución del número de telespectadores (en miles) en las televisiones estudiadas.

\section{El Estudio General de Medios: método de medición de audiencias en constante evolución}

El Estudio General de Medios (EGM) es el sistema de medición vigente y predominante durante el periodo de estudio. Utilizando la técnica de la encuesta, se procede mediante cuestionarios- a realizar las entrevistas sobre el recuerdo de la víspera.

El EGM nace en 1968 promovido por anunciantes, agencias y medios. En 1975 se constituye en sociedad anónima pasando de estar financiado por las agencias de publicidad a depender de todos los organismos implicados en su uso, intentando de este modo, asegurar su objetividad (Huertas, 1998).

Posteriormente, en 1988 se transforma en Asociación constituyéndose formalmente la Asociación para la Investigación de los Medios de Comunicación (AIMC), actualmente vigente. Desde entonces, tal y como figura en sus Estatutos, la AIMC(1988b) carece de ánimo de lucro.

Con el paso de los años el sistema se ha ido perfeccionando a través de constantes revisiones y actualizaciones. Esta evolución tiene que ver por un lado con la adjudicación del EGM a distintos organismos de investigación (ECO: 1968 a 1972, 1978 a 1981 y 1983 hasta la actualidad; Metra Seis: 1975 1978; Saltés: 1982) y a la propia mejora del sistema. Si bien, ha permanecido el esquema de trabajo base de la investigación que contemplando los siguientes parámetros: características técnicas, universo, muestra, trabajo de campo, inspección, cuestionario y contenidos de la información, procesado de datos, resultados, usos y utilidades (Ávila, 1999).

El EGM está basado en la teoría estadística de las muestras, un universo aleatorio estratificado donde todos los miembros de la población tienen una probabilidad conocida de estar en la muestra (Jauset, 2000).

Tal y como se estructura desde 1978, el EGM se perfila como un estudio de audiencia de medios, ya que además de recopilar los datos sobre televisión, obtiene datos sobre diarios, suplementos dominicales, revistas, radio y cine. De este modo, se mide el comportamiento de individuos de 15 años y más, residentes en núcleos urbanos. A partir de 1984 comienzan a recoger datos relativos a las televisiones autonómicas; en 1985 se introduce la programación específica del día anterior por segmentos horarios (hasta entonces los datos disponibles hacen referencia a bloques horarios); y en 1988 se amplía el universo incorporando a los individuos de 14 años y a aquellos residentes en poblaciones de más de 200 habitantes, accediendo así a los individuos de núcleos rurales. Toda esta información se recogía durante 2 semanas por ola ${ }^{7}$ hasta que

7 Se realizan tres oleadas de recogida de datos al año. 
en 1987 se establece un período de 8 semanas para proceder a la recogida de datos. Una vez recogidos los datos, la grabación y su procesado lo realizaba ODEC en su sede de Gandía, en la provincia de Valencia (Ávila, 1999).

\section{Evolución de la audiencia en televisión (1983-1990): de las audiencias cautivas a las audiencias compartidas}

A lo largo de estos años, existe una clara tendencia al alza en el número de telespectadores de las televisiones, aún ciertamente escasas, que operan en el mercado español. De entre todas ellas destaca claramente TVE1. El primer canal de Televisión Española sigue viviendo una edad dorada a pesar de compartir escenario no sólo con TVE2 si no también, a partir de 1983, con los primeros canales autonómicos públicos. Según el EGM (véase Gráfico 1), entre 1983 y 1990 el número total de telespectadores en España se incrementa desde los 21.290.000 hasta los 27.605.000.

Gráfico 1: Evolución de la audiencia de televisión en España (1983-1990)

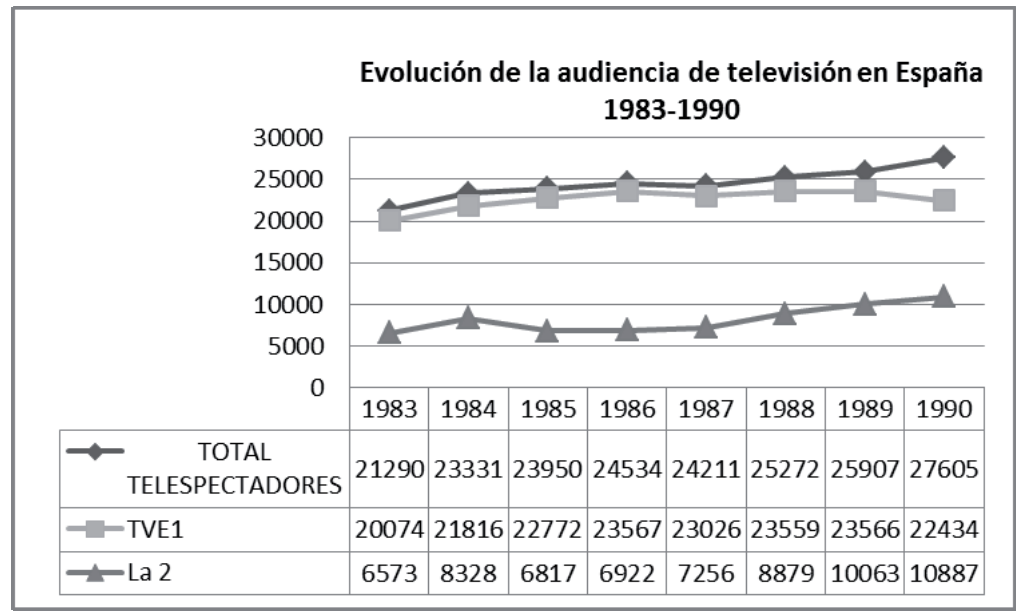

Fuente: Elaboración propia con datos del EGM/AIMC

Si observamos los datos relativos a Televisión Española podremos concluir que TVE1 es la opción de programación preferida por los telespectadores españoles, si bien la presencia de otras opciones de televisión alternativas son ciertamente escasas. De los poco más de 20 millones alcanzados en 1983, inicia una tendencia al alza hasta alcanzar en 1989 su punto de máximo esplendor, superando los 23 millones de telespectadores. El dato referido a 1990 deja ya entrever el final de esa edad dorada de audiencias cautivas y el comienzo de una etapa de progresivo descenso de las audiencias que coincide con la desregulación del sector de la televisión en España y el inicio de emisiones de las primeras televisiones privadas en la Neotelevisión: Antena 3, Telecinco y Canal + .

A partir de entonces, las cadenas privadas inauguran una etapa caracterizada por el progresivo incremento de sus audiencias y por la consolidación de su cuota de mercado, hecho que repercutirá inexorablemente, sobre las audiencias de las televisiones 
públicas. Esto es especialmente significativo en el caso de TVE, acostumbrada a unas audiencias que nunca más volverá a alcanzar.

Los datos relativos a la cuota de mercado de las televisiones españolas en 1990 (véase Gráfico 2), permiten constatar la aún importante cifra alcanzada por los canales de Televisión Española, cercana al 73\% y especialmente de TVE1 $(52,4 \%)$ pero significativa también en el caso del segundo canal con un 20,2\%. Asimismo hemos podido corroborar la ya significativa presencia de las televisiones autonómicas que alcanzan conjuntamente el $16,4 \%$ y la aún tímida presencia de las televisiones privadas en abierto con un $10,5 \%$, destacando Telecinco con el $6,5 \%$ y en menor medida Antena $3 \mathrm{TV}$ con el 3,7\%.

Gráfico 2: Mercado de la televisión en España (1990)

\section{Mercado de la televisión en España 1990}



- TVE1

- La 2

- Telecinco

- Antena 3

- Canal $+(\mathrm{a})$

Autonóm.

- Otras

Fuente: Elaboración propia con datos de Kantar Media

\section{Audiencia y programación en el comienzo del proceso de democratización de Televisión Española: el ocaso del modelo paleotelevisivo \\ 5.1. Estructura de la audiencia en TVE}

Los datos relativos a la estructura de la audiencia en los dos canales de Televisión Española que pasamos a comentar, nos permitirán describir algunos rasgos característicos que hemos elaborado tomando como base los datos facilitados por el EGM sobre la muestra de un año (noviembre de 1982 a octubre de 1983) que se resume en la Tabla 1.

Tabla 1: Estructura de la audiencia en TVE (noviembre de 1982 a octubre de 1983)

\begin{tabular}{|ccccccccccccccc|}
\hline & Sexo & \multicolumn{1}{c|}{ Edad } & \multicolumn{1}{c|}{$\begin{array}{c}\text { Clase } \\
\text { social }\end{array}$} \\
\hline & H & M & $15 / 18$ & $19 / 24$ & $25 / 34$ & $35 / 44$ & $45 / 54$ & $55 / 64$ & $\begin{array}{c}65 / \\
\text { más }\end{array}$ & Alta & $\begin{array}{c}\text { Media } \\
\text { alta }\end{array}$ & $\begin{array}{c}\text { Media } \\
\text { media }\end{array}$ & $\begin{array}{c}\text { Media } \\
\text { baja }\end{array}$ & Baja \\
TVE1 & 49 & 51 & 10,4 & 11,5 & 17,5 & 14,8 & 14 & 14,5 & 17,4 & 5,5 & 15,7 & 25,8 & 20,5 & 32,4 \\
TVE2 & 47,8 & 52,2 & 10,8 & 12,8 & 21,4 & 15,8 & 13,3 & 12,9 & 13,1 & 6,5 & 17,9 & 27,4 & 21,2 & 27 \\
\hline
\end{tabular}

Fuente: Elaboración propia con datos del EGM. Población: 26.473.000 / Records: 18.367 
La audiencia de TVE1 es mayoritariamente femenina, un 51\% frente al 49\% restante de hombres.

Respecto a la edad de sus telespectadores, destacan significativamente dos franjas de edad: la comprendida entre los 25 y 34 años que supone un 17,5\% y la de 65 años y más con un $17,4 \%$. A corta distancia le siguen aquellas personas con una edad comprendida entre los 35 y 44 años $(14,8 \%)$, entre los 55 y 64 (14,5\%) y entre 45 y 54 años $(14 \%)$.

Por el contrario, hay que destacar la menor importancia de los públicos más jóvenes, alcanzando un 10,4\% los jóvenes de 15 a 18 años y un 11,5\% los de 19 a 24 años.

En lo que se refiere a la clase social destaca mayoritariamente la clase baja con un $32,4 \%$, seguida de la media-media con un $25,8 \%$ y la media-baja con el $20,5 \%$.

En el polo opuesto encontraríamos a la clase alta que supone a lo largo del periodo de estudio el 5,5\% de la audiencia y la media-alta que alcanza el 15,7\%.

La clase baja y media baja supone -por tanto- el 52,9\%, una mayoría de la audiencia de TVE1 frente al 21,2\% que alcanza la suma de la clase alta y media-alta.

En el caso del segundo canal de Televisión Española, también podemos hablar de una audiencia mayoritariamente femenina pero ligeramente superior a la obtenida por TVE1, ya que alcanza una cifra superior al $52 \%$ frente al $47,8 \%$ de hombres.

Respecto a la edad de la audiencia, hemos comprobado que se mantiene la importancia de personas de entre 25 y 34 años, si bien esta franja de edad se refuerza significativamente en el caso de TVE2 ya que alcanza el 21,4\%. A considerable distancia le siguen en orden de importancia la franja de 35 a 44 años $(15,8 \%)$, la de 45 a $54(13,3 \%)$, la de 65 años y más con un 13,1\% y finalmente la de 55 a 64 años con el 12,9\%.

Resulta llamativo el hecho de que todas las franjas de edad consideradas más significativas se reducen ligeramente en el caso de TVE2 respecto a las cifras de TVE1 y es especialmente apreciable la reducción de las personas de mayor edad.

En lo que se refiere a las franjas de edad con menos presencia en el cómputo de audiencia del segundo canal de TVE, se observa una tendencia similar a la detectada en TVE1 ya que son, una vez más, los públicos más jóvenes los menos consumidores de TVE2, si bien las cifras son ligeramente superiores a las alcanzadas en el primer canal: $10,8 \%$ para las audiencias de 15 a 18 años y $12,8 \%$ para las de 19 a 24 años.

Finalmente, en lo que respecta a los datos relativos a la clase social de la audiencia de TVE2, observamos ligeras variaciones respecto a los obtenidos para el primer canal, destacando la clase media-media como la de mayor presencia con un $27,4 \%$, seguida de la baja con un $27 \%$ y de la media-baja con un $21,2 \%$.

Es por tanto la clase media-media la que obtiene cifras mayores en el caso de TVE2 y un porcentaje superior al que alcanzó en TVE1.

La clase baja si bien mantiene la segunda posición, su porcentaje disminuye respecto al alcanzado en TVE1 ( $27 \%$ frente al $32,4 \%)$.

Las clases alta y media-alta son una vez más las que obtienen cifras inferiores en TVE2 pero se refuerzan respecto a las alcanzadas en TVE1 (6,5\% frente a 5,5\% para la clase alta y $17,9 \%$ frente a $15,7 \%$ para la media-alta.

La clase baja y media-baja suponen en TVE2 el 48,2\%, no llegando por tanto a la mitad de la audiencia (en TVE1 la superaba) y la clase alta y media-alta alcanzan el 
24,4\% reforzando de este modo, su presencia en el segundo canal de Televisión Española. La explicación a esta oscilación en los datos habría que buscarla en el modelo de programación y en la tipología de programas ofertados por los dos canales de TVE.

\subsection{El modelo de programación generalista en TVE}

Durante el periodo de tiempo analizado, Televisión Española continúa apostando por un modelo de programación generalista con un claro corte cultural y educativo, (especialmente relevante en el caso de TVE2 pero también presente en el primer canal).

Este modelo de gestión de los contenidos se pone en práctica en TVE 1, diseñando de lunes a viernes un esquema de programación horizontal, que viene avalado por los hábitos de vida uniformes de la audiencia potencial y que se materializa programando todos los días a la misma hora el mismo programa, una estrategia denominada stripping que los profesionales suelen utilizar con objeto de conseguir la fidelización de las audiencias. Este esquema de programación horizontal está presente en todas las franjas horarias salvo en la de la noche, donde se insertan las grandes apuestas diversificando los contenidos, con la excepción del Telediario que permanece estable. Pero salvo esta excepción, hemos constatado la presencia en la franja nocturna de una diversidad de programas: concursos como el mítico Un, dos, tres, programas de entrevistas como Españoles presentado por Victoria Prego o el Buenas noches de Mercedes Milá, cine en Sesión de Noche o series como Anillos de oro protagonizada por una pareja de abogados especializados en causas matrimoniales, rodada poco después de legalizarse el divorcio en España, una pequeña muestra de cómo los cambios políticos y sociales vividos tras la dictadura franquista, comienzan a hacerse presentes en los contenidos televisivos de TVE.

La programación generalista de Televisión Española a la que aludíamos, se organiza durante el fin de semana diseñando un esquema vertical. Los profesionales organizan singularmente la programación para el sábado y para el domingo porque los hábitos de vida de la audiencia durante esos días también son distintos. Y dado el interés que tradicionalmente ha habido en España por el fútbol, no puede extrañarnos la importancia de este deporte en la programación de tarde los sábados y en las franjas de tarde y noche de los domingos. Pero también otras tipologías de programas están presentes durante el fin de semana como veremos a continuación.

En el caso del segundo canal, el esquema de programación horizontal tan solo se produce entre las 19 y las 20 horas momento en el que se emiten espacios de corta duración como Informativo 2, las sesiones de aerobic con Eva Nasarre en Puesta a punto o los Cursos de inglés y a las 21 horas en que se emite Hoy en la 2. Y durante el fin de semana, el diseño del modelo de programación sobre el que se trabaja es muy similar al ya comentado respecto de TVE1, se trabaja sobre un esquema de programación vertical por los motivos ya comentados.

\subsection{Análisis del comportamiento de las audiencias}

Desde el punto de vista cuantitativo, las audiencias de TVE1 son claramente superiores a las alcanzadas por TVE2, unos 20 millones frente a 6,5 (véase Gráfico 1), de ahí que podamos considerarlo el canal estrella. 
Es importante destacar el significativo rendimiento en antena de la programación emitida en la franja de la noche (21 a 24h) en los dos canales de Televisión Española. El horario de máxima audiencia o prime time ha sido y continúa siendo en la actualidad, el de mayor relevancia, precisamente por ser el momento de la jornada preferido por los telespectadores para ver la televisión (contrariamente a lo que sucede con otros medios de comunicación como por ejemplo la radio) y el que mejor se adapta a los hábitos de vida de la mayoría, de ahí su relevancia.

Tabla 2: Audiencia en TVE por franjas horarias (1983-1990)

\begin{tabular}{|c|c|c|c|c|c|}
\hline \multirow[t]{2}{*}{1983} & \multicolumn{5}{|c|}{1990} \\
\hline & TVE 1 & TVE 2 & & TVE 1 & TVE 2 \\
\hline- & - & - & $\begin{array}{l}\text { Madrugada } \\
(4-8)\end{array}$ & 0,3 & 0,1 \\
\hline $\begin{array}{l}\text { Mañana } \\
(10-14)\end{array}$ & 3,3 & - & $\begin{array}{l}\text { Mañana } \\
(8-13,30)\end{array}$ & 10,6 & 3,2 \\
\hline $\begin{array}{l}\text { Sobremesa } \\
(14-17)\end{array}$ & 39,8 & 0,6 & $\begin{array}{l}\text { Sobremesa } \\
(13,30-17)\end{array}$ & 49,3 & 10,4 \\
\hline $\begin{array}{l}\text { Tarde } \\
(17-21)\end{array}$ & 35,1 & 7,9 & $\begin{array}{l}\text { Tarde } \\
(17-20)\end{array}$ & 16,2 & 12,5 \\
\hline $\begin{array}{l}\text { Noche } \\
(21-24)\end{array}$ & 63,9 & 21,3 & $\begin{array}{l}\text { Noche } \\
(20-4)\end{array}$ & 49,8 & 23,5 \\
\hline
\end{tabular}

Fuente: Elaboración propia con datos del EGM/AIMC

Tal y como se refleja en la Tabla 2, TVE1 obtiene en esta franja horaria una audiencia cercana al $64 \%$, seguida de la sobremesa con una cifra próxima al $40 \%$ y de la franja horaria de la tarde con un $35,1 \%$.

El éxito de la programación de la noche con cifras de vértigo solo se explica en un contexto de monopolio de las televisiones públicas y por tanto de audiencias cautivas.

Es importante destacar la aún escasa importancia de la franja de la mañana, una franja horaria de emisiones discontinuas, con cifras mínimas que comenzaba retransmitiendo la denominada Carta de ajuste con música ambiental, en horarios diversos, que avanzaba el inicio de la programación también en otras franjas horarias.

Revisando los periódicos de la época hemos comprobado que estas emisiones matinales, cuando tenían lugar (especialmente fines de semana y ante la cobertura informativa de eventos), comenzaban entre las 8,30 y las 11, dependiendo de las necesidades de programación y a continuación se podía emitir programación de servicio público como la Misa, retransmisiones de eventos como la visita a España realizada por el Papa Juan Pablo II en noviembre de 1982 o programación cultural como documentales, conciertos, etc.

La sobremesa es otro bloque horario significativo en las audiencias del primer canal de Televisión Española en el que los días laborables, además de la programación regional y el Telediario, se programan telefilmes como Thomas y Sarah o magazines como La tarde presentado por Pepe Navarro. Durante los fines de semana tiene cabida la programación de espacios vinculados con la actualidad como Revista de toros o Las Cortes de España y programación infantil y juvenil como Los pitufos, la serie Fama y programación cinematográfica para toda la familia con Primera sesión. 
En el caso del segundo canal de TVE, las cifras de audiencia se diferencian claramente de las alcanzadas por su canal "hermano". Si bien, la franja horaria de la noche sigue siendo la opción preferida por su audiencia, como cabía esperar, el porcentaje registrado se sitúa en el 21,3\%, una cifra muy alejada de las cotas del primer canal.

En esta franja de máxima audiencia encontramos mayoritariamente una programación cultural y de servicio público. Programas como El melodrama, Música y músicos, La buena música, La edad de oro, Teatro Real, Cine club series dramáticas como la adaptación televisiva de Los gozos y las sombras o el programa Si yo fuera presidente presentado por Fernando García Tola en el que la audiencia podía formular quejas o peticiones a políticos, sindicatos o líderes sociales.

Pero además de los datos esperados, derivados de los hábitos de vida y de las preferencias horarias de consumo televisivo de las audiencias, hay que añadir otros factores que contribuyen a explicar los datos que se reflejan en la Tabla 2, en la que se pone de manifiesto la audiencia mínima $(0,6 \%)$ en la sobremesa y con un ligero incremento $(7,9 \%)$ en la franja de la tarde:

- Menor número de horas de emisión: de lunes a jueves las emisiones se inician a las siete de la tarde con el Informativo 2 de diez minutos y concluyen hacia las doce de la noche. Ello explica la inexistencia de datos de audiencia en la franja de la mañana.

- Horarios de emisión diferenciados de viernes a domingo. Sólo durante estos días, las emisiones se inician en torno a las $14,30 \mathrm{~h}$ y concluyen hacia medianoche.

\section{Audiencia y programación en el umbral de la Neotelevisión}

\subsection{Estructura de la audiencia en TVE}

Tomando como base los datos facilitados por el EGM relativos al periodo de estudio comprendido entre febrero y noviembre de 1990, podemos trazar algunas directrices sobre el comportamiento de la audiencia de TVE en el umbral de la desregulación, momento en que se inicia una nueva edad televisiva, la Neotelevisión y sus novedosas prácticas profesionales hasta entonces innecesarias para las televisiones públicas.

Tabla 3: Estructura de la audiencia en TVE (febrero a noviembre de 1990)

\begin{tabular}{|c|c|c|c|c|c|c|c|c|c|c|c|c|c|c|}
\hline & Sexo & & Edad & & & & & & & $\begin{array}{l}\text { Clase } \\
\text { social }\end{array}$ & & & & \\
\hline & $\mathrm{H}$ & $M$ & $14 / 19$ & $20 / 24$ & $25 / 34$ & $35 / 44$ & $45 / 54$ & $55 / 64$ & $\begin{array}{l}\text { 65/ } \\
\text { más }\end{array}$ & Alta & $\begin{array}{l}\text { Media } \\
\text { alta }\end{array}$ & $\begin{array}{l}\text { Media } \\
\text { media }\end{array}$ & $\begin{array}{l}\text { Media } \\
\text { baja }\end{array}$ & Baja \\
\hline TVE1 & 46 & 54 & 12,4 & 9,6 & 17,9 & 14,9 & 13,1 & 14,5 & 17,5 & 4 & 11,5 & 36,3 & 29 & 19,2 \\
\hline TVE2 & 55,8 & 44,2 & 13 & 10,3 & 19,4 & 15 & 12,5 & 13,7 & 16,1 & 4,3 & 13,1 & 38,6 & 28,2 & 16,8 \\
\hline
\end{tabular}

Fuente: Elaboración propia con datos del EGM. Población: 31.575.000/Entrevistas: 43.946

Hemos detectado una vez más, la polarización de la audiencia del primer canal de TVE si bien, los datos ponen de manifiesto el predominio de telespectadores femeninos: un $54 \%$ frente al $46 \%$ de hombres.

Respecto a la edad, destacamos nuevamente la franja de edad comprendida entre los 25 y 34 años que ocupa la primera posición con un 17,9\%, seguida de las perso- 
nas de 65 años y más (17,5\%), las personas de entre 35 y 44 años $(14,9 \%)$, de 55 a 64 $(14,3 \%)$ y las de 4 a 54 años $(13,1 \%)$.

Por el contrario, la audiencia menos numerosa en TVE1 la constituye los jóvenes. Así, aquellos con una edad comprendida entre los 20 y los 24 años representan el $9,6 \%$ de la audiencia y los de 14 a 19 años alcanzan una cifra ligeramente superior, el $12,4 \%$.

La clase media-media es la más numerosa en el primer canal de TVE alcanzando el $36,3 \%$, seguida de la media-baja con un $29 \%$ y a considerable distancia, la clase baja que representa el 19,2\% de su audiencia. La clase alta, por el contrario, es la menos representativa obteniendo tan solo el $4 \%$ junto con la clase media-alta que alcanzó el $11,5 \%$.

Pero, si bien la clase media-media es la más representativa, si sumamos los porcentajes de las clases media-baja y baja veríamos que representan el $48,2 \%$ de la audiencia de TVE1 frente a la clase alta y media-alta que representan tan solo el 15,5\%.

Respecto a la estructura de la audiencia del segundo canal de Televisión Española cabría señalar en primer lugar, su perfil mayoritariamente masculino con un porcentaje del 55,8\%. Es por tanto un rasgo diferencial entre los dos canales de TVE. Recordemos que TVE1 en ese periodo de estudio, está dominado mayoritariamente por audiencia femenina.

La estructura de la audiencia en función de las franjas de edad sigue el esquema, en gran medida similar al de TVE1 si bien, se refuerzan algunos grupos como el comprendido entre los 25 y los 34 años, el más numeroso en TVE2 que alcanza el 19,4\%, los individuos de 20 a 24 años (10,3\%) y los jóvenes de 14 a 19 (13\%) que a pesar de reforzarse respecto a TVE1, continúan siendo las audiencias menos numerosas también en el caso del segundo canal. Los individuos de 45 a 54 años reducen ligeramente su presencia en TVE2 durante el periodo estudiado.

Y ya para concluir esta aproximación a la estructura de la audiencia de TVE2 cabría añadir los datos relativos a la clase social de la audiencia. Las bases de datos manejadas ponen de manifiesto rasgos diferenciales entre los dos canales de Televisión Española. De hecho, TVE2 refuerza notablemente las cifras porcentuales relativas a la clase media-media $(38,6 \%)$, a la clase media-alta $(13,1 \%)$ y también las relativas a la clase alta $(4,3 \%)$. Por el contrario, se reducen respecto al primer canal la clase media-baja $(28,2)$ y la baja $(16,8 \%)$, sumando ambas el $45 \%$. En cualquier caso, las clases media-media, media-baja y baja siguen siendo las de mayor notoriedad en la audiencia de TVE2.

\subsection{Modelos de programación cada vez más diferenciados}

El modelo de programación vigente durante el presente periodo de estudio continúa siendo el generalista si bien, empiezan a perfilarse algunas modificaciones que avanzan estrategias de actuación que se generalizarán con el comienzo de emisiones de las cadenas de televisión privadas.

La programación de divulgación cultural, la programación educativa y especialmente, la programación deportiva, se traspasa progresivamente a la parrilla de TVE2 de ahí la consolidación del target masculino, mientras que La Primera, sin erradicar 
totalmente la programación cultural, mantiene y refuerza aquella programación que mejor se rentabiliza desde el punto de vista de los índices de audiencia.

En términos generales, TVE1 mantiene de lunes a viernes el diseño de la parrilla horizontal, con inicio de emisiones a las 8 de la mañana, salvo en 3 periodos horarios: de $13,30 \mathrm{~h}$ a $14 \mathrm{~h}$, en la franja de acceso a la sobremesa; de 17,30h a $18 \mathrm{~h}$ (prolongándose los viernes hasta las 19,30h aproximadamente); y en el prime time, la franja horaria que se inicia tras el Telediario2, en la que se insertan las grandes apuestas de programación de la cadena.

Por el contrario, durante el fin de semana, se diseña la parrilla siguiendo un esquema de programación vertical, por los motivos ya explicados en el análisis del periodo de estudio anterior.

Por su parte, el esquema de programación de TVE2 se modifica sustancialmente respecto del comentado para el período noviembre de 82 a octubre de 83 , dado que se generaliza de lunes a domingo el inicio de emisiones a las 8 de la mañana, concluyendo hacia las 2,30 de la madrugada. Por ello podríamos establecer un primer rasgo característico como es el notable incremento del número de horas de programación hasta alcanzar las 18 horas y media diarias, frente a las 5 diarias de lunes a jueves 0 las 9 horas y media de viernes a domingo durante 1983.

De lunes a viernes se diseña una parrilla de programación horizontal de 8 a $12 \mathrm{~h}$ y de 13 a $20 \mathrm{~h}$ aproximadamente (con excepción de los viernes de 15,30 a 16,30h), estableciendo para el resto de periodos horarios un esquema vertical.

Durante el fin de semana el diseño de la parrilla es mayoritariamente vertical con excepción de la franja de 8 a 9 de la mañana con programación musical y de 14,30 a 14,35 y de 20 a $20,10 \mathrm{~h}$ en que se emiten breves espacios de noticias.

\subsection{Análisis del comportamiento de las audiencias de TVE en el comienzo de la desregulación}

Una vez más, partimos de un rasgo cuantitativo diferencial de las audiencias de los dos canales de Televisión Española dado que en 1990 TVE1 alcanza una cifra de 22.434.000 telespectadores frente a los 10.887.000 de TVE2 (véase Gráfico 1). Por tanto, el primer canal duplica ampliamente en audiencia al segundo canal de TVE.

En este periodo de estudio llama especialmente la atención la distribución de audiencia en TVE1 dado que el porcentaje alcanzado en el horario de noche ${ }^{8}(49,8 \%)$ es muy similar al de la sobremesa (49,3\%) y esto es algo excepcional. Estamos, por tanto, ante las dos franjas horarias preferidas por la audiencia de Televisión Española.

- El horario de noche, que de nuevo se inicia tras el Telediario2 se nutre de contenidos (ficción, información y entretenimiento) de éxito, capaces de intentar rivalizar con la amenaza que supone el inicio de emisiones de las televisiones privadas. Así, nos encontramos con concursos como El precio justo, una adaptación del formato estadounidense The price is right procedente de la CBS que en España estuvo inicialmente presentado por Joaquín Prat. También con programas no vistos hasta entonces

8 Hay que hacer constar que durante este periodo de estudio, el EGM establece modificaciones en la cuantificación de la franja horaria de la noche, incluyendo no solo el prime time sino también el periodo comprendido entre la medianoche y las 4 de la madrugada. 
en las televisiones españolas como Hablemos de sexo, emitido entre marzo y noviembre de 1990 que contó con la presentación de la doctora Elena Ochoa y la dirección de Narciso Ibáñez Serrador. Se trataba de un espacio en el que por primera vez se abordan en televisión temas relacionados con la sexualidad humana. Programas con vídeos domésticos elaborados por los telespectadores como Vídeos de primera. Un formato de bajo coste que se rentabiliza muy bien en antena debido a las audiencias que alcanza.

Espacios de ficción seriada entre los que cabe destacar sitcoms como Las chicas de oro o Juzgado de guardia capaces de aglutinar y fidelizar semana tras semana importantes cifras de audiencia; o las soap opera de lujo, cuyo principal exponente es Falcon Crest, la serie revelación que causó furor en aquella época. Asimismo mantienen su presencia los programas de entrevistas y debates como Un día es un día presentado por Ángel Casas que finalizaba con un streaptease en torno a la media noche; el cine que continúa presente en el horario de noche con espacios como Sesión de noche, Estrenos TV, Sábado cine o Domingo cine o los espacios informativos como el Telediario 2, programas de reportajes de actualidad como Informe Semanal y espacios de grandes documentales periodísticos cuya muestra es En Portada.

- En la sobremesa de TVE1, además de destacar-de lunes a viernes- la importancia de la programación informativa con el Telediario 1 y El Tiempo y, en menor medida los informativos elaborados por los centros regionales que compiten abiertamente con los de las televisiones autonómicas, hay que hacer referencia al género revelación en los primeros años noventa: las telenovelas latinoamericanas cuyo exponente en el periodo analizado es Cristal, serie cuyo gran éxito de audiencia -en ocasiones llega a superar los 8 millones de telespectadores- explica en parte los datos globales de audiencia, ya comentados, de esta franja horaria. Durante el fin de semana, la sobremesa incluye, además de los espacios fijos de programación informativa, media hora de dibujos animados con Los Fruitis o Las mil y una América y la programación cinematográfica.

- La franja horaria de la tarde obtiene un $16,2 \%$ de audiencia lo que la convierte en la tercera franja horaria del ranking. Los días laborables, además de los magazines como Esta es mi casa, es relevante la programación infantil con espacios como Cajón desastre o Los mundos de Yupi y la programación juvenil con series como Corrupción en Miami, Kung Fu o Young Riders. Durante el fin de semana permanece la programación familiar con cine y concursos como El tiempo es oro y contenidos infantiles con Club Disney o La hora Warner.

- En lo que se refiere a la franja de la mañana (8-13,30h) cabría destacar que si bien no es un periodo horario de grandes audiencias, se intenta fidelizar de lunes a viernes targets delimitados, especialmente amas de casa y jubilados, con series como Santa Bárbara o La Isla. También es importante hacer referencia al espacio informativo Buenos días, primer espacio matinal de estas características con el que desde 1986 se inician las emisiones de esta franja horaria.

Durante el fin de semana, por el contrario, cobra importancia la programación infantil, muestra de ello es la presencia en la parrilla de espacios como Sopa de gansos, No te lo pierdas, Pista de estrellas o 3,2,1 ... contacto, y la programación religiosa con 
la retransmisión de la Misa o Pueblo de Dios. Y finalmente cabría mencionar la franja de la madrugada con audiencias mínimas en la que tienen cabida las redifusiones.

La audiencia del segundo canal_de Televisión Española se concentra mayoritariamente en la franja de la noche donde se alcanza una cifra porcentual del 23,5.

Si observamos el esquema de programación coincidente con el periodo de estudio comprobaremos que de lunes a viernes, en esta franja además del espacio informativo TVE2. Noticias de 15 minutos de duración, se emite fútbol, cine, el espacio informativo de documentales Documentos TV, series en Relatos TV, series documentales como Los felices 80 presentado por Xabier Elorriaga sobre hechos de interés acaecidos en la mencionada década o programas de tertulia como Tribunal popular.

Durante el fin de semana, la programación se completa con grandes dosis de cine, programas musicales como Qué noche la de aquel año presentado por el cantante Miguel Ríos, series como Juncal dirigida por Jaime de Armiñán o programas de variedades como Sara y punto con la artista Sara Montiel como protagonista.

A partir de la medianoche tienen cabida otro tipo de espacios culturales experimentales con audiencias más minoritarias como Metrópolis, series como El autoestopista, series documentales como Testigos del siglo $X X$ y nuevamente cine a través de espacios como Filmoteca TV, Última sesión o Minicine.

Las demás franjas horarias obtienen unas cifras de audiencia inferiores. La franja de la tarde (17-21) alcanza el 12,5\% con una importante presencia del cine, espacios culturales y deportivos en la programación emitida de lunes a viernes. La notoriedad de la programación deportiva y el cine (en menor medida) se mantienen también durante el fin de semana y copan la programación de este periodo horario.

La sobremesa (13,30-17) que consigue el $10,4 \%$ de la audiencia, incluye programación musical con espacios como Klip, documentales, series como Historias de cada día y dos breves informativos de cinco minutos a las $14,30 \mathrm{~h}$ y a las $16,30 \mathrm{~h}$.

Durante el fin de semana, la programación de sobremesa de TVE2 es exclusivamente deportiva, si bien los sábados se añade a las 14,30h el espacio de contenido religioso Últimas preguntas.

Finalmente mencionar que la franja de la mañana (8-13,30h) alcanza una audiencia escasa, tan solo el 3,2\%. Las emisiones de esta franja de lunes a viernes comienzan y concluyen con dibujos animados, dos horas de contenidos educativos ( $T V$ educativa) y el magazine La hora de...

Durante el fin de semana coexisten los espacios culturales y educativos con los deportivos (domingos a partir de las 12) y la franja de la madrugada apenas registra audiencia.

\section{Conclusiones}

El estudio realizado a lo largo de estas páginas nos ha permitido delimitar algunos rasgos definitorios de las audiencias y el modelo de programación en Televisión Española en una etapa histórica de monopolio de las televisiones públicas y hegemonía incuestionable de la televisión estatal, especialmente de TVE1, que a partir de 1990 perderá progresivamente su centralidad compartiendo protagonismo con las televisiones privadas, hecho de capital importancia que anuncia el final de una etapa dorada 
para TVE y el paso de las audiencias cautivas a las audiencias compartidas, con todo lo que ello implica desde el punto de vista de la rentabilidad y de los contenidos ofertados. Desde entonces, será imposible para TVE volver a alcanzar audiencias superiores a los 20 millones de telespectadores.

Esta hegemonía de audiencia de TVE1 contrasta con los datos de audiencia alcanzados por el segundo canal que, si bien, durante el periodo estudiado incrementa progresivamente sus audiencias en ningún momento llega a aproximarse a los datos de su canal hermano, de hecho ya en esta época irá afianzando su rol de canal de minorías para audiencias alternativas cuya explicación debemos buscarla en el diseño del modelo de programación y en los contenidos ofertados.

Durante este periodo, los dos canales de Televisión Española apuestan por un modelo de programación generalista en el que tiene cabida el entretenimiento pero con altas dosis de contenidos culturales, educativos y de servicio público, estos atributos progresivamente se van transfiriendo hacia la oferta programática del segundo canal que -además- se refuerza con contenidos deportivos, de ahí la evolución del perfil de audiencia -mayoritariamente masculina- que alcanza TVE2 hacia 1990. Recordemos que en términos generales, es la audiencia femenina la mayoritaria en Televisión Española.

Los contenidos estrella, las grandes apuestas están presentes mayoritariamente en la franja horaria de la noche, especialmente en el denominado prime time, donde la televisión aglutina las mayores cifras de audiencia. Sin embargo se ha constatado cómo un contenido estrella (hemos aludido al caso de la telenovela venezolana Cristal) que se programe en otra franja horaria -la sobremesa- puede intervenir en los hábitos de consumo televisivo de las personas y multiplicar las datos de audiencia equiparándolos a los del prime time.

Estamos pues ante dos canales generalistas en los que cabe la información, la cultura y el entretenimiento de calidad pero con objetivos de programación diversos, lo cual repercute directamente sobre la cuantificación y la estructura de la audiencia y permite perfilar unos canales con contenidos y audiencias complementarias.

\section{Referencias}

AIMC (1988a): Egm 30. Madrid, AIMC

AIMC (1988b): Estatutos: http://www.aimc.es/-Estatutos-y-Reglamento-.html [fecha de consulta 17 de junio de 2013]

ÁVILA ARRABAL, Laura (1999): Del EGM a la AIMC. Evolución histórica (19681998). Madrid, AIMC.

BUSTAMANTE, Enrique (2002): “Televisión: errores y frenos en el cambio digital”, en BUSTAMANTE, Enrique (coord.): Comunicación y cultura en la era digital. Industrias, mercados y diversidad en España. Barcelona, Gedisa, pp. 213-264

CORTÉS, José Ángel (1999): La estrategia de la seducción. Pamplona, Eunsa.

ECO, Umberto (1986): La estrategia de la ilusión. Barcelona, Lumen.

FERNÁNDEZ ALONSO, Isabel y SANTANA, Fernanda (2000): Estado y medios de comunicación en la España democrática. Madrid, Alianza. 
GUIMERÁ, Josep Àngel y BLASCO, José Joaquín (2012): "La formación histórica del sistema televisivo autonómico público (1982-2011)", en MIGUEL, Juan Carlos y CASADO, Miguel Ángel: Televisiones autonómicas. Evolución y crisis del modelo público de proximidad. Barcelona, Gedisa, pp. 33-57.

HUERTAS BAILÉN, Amparo (1998): Cómo se miden las audiencias en televisión. Barcelona, CIMS.

HUERTAS BAILÉN, Amparo (2002): La audiencia investigada. Barcelona, Gedisa.

JAUSET, Jordi A. (2000): La investigación de audiencias en televisión. Fundamentos estadísticos. Barcelona, Paidós.

KANTAR MEDIA (2011): Anuario de las audiencias de televisión. Madrid, CD Rom

Ley 4/1980 de 10 de enero, Estatuto de la Radio y la Televisión, BOE no 11 de 12 de enero, http://www.boe.es/diario_boe/txt.php?id=BOE-A-1980-724 [fecha de consulta 16 de mayo de 2013]

Ley 10/1988 de 3 de mayo, de Televisión Privada BOE 108 de 5 de mayo, http://www.boe.es/buscar/doc.php?id=BOE-A-1988-11073 [fecha de consulta 16 de mayo de 2013]

ROEL, Marta (2006): "La televisión en la democracia española (1982-2005). Dinámicas de los modelos de gestión de contenidos", en ARROYO, María y ROEL, Marta (coords.): Los medios de comunicación en la democracia (1982-2005). Prensa, radio y televisión. Internet y grupos de comunicación. Madrid, Fragua, pp.91-159.

\section{Anexos}

Esquema de programación de TVE para el periodo de estudio noviembre de 1982 a octubre de 1983 (AIMC 1988a).

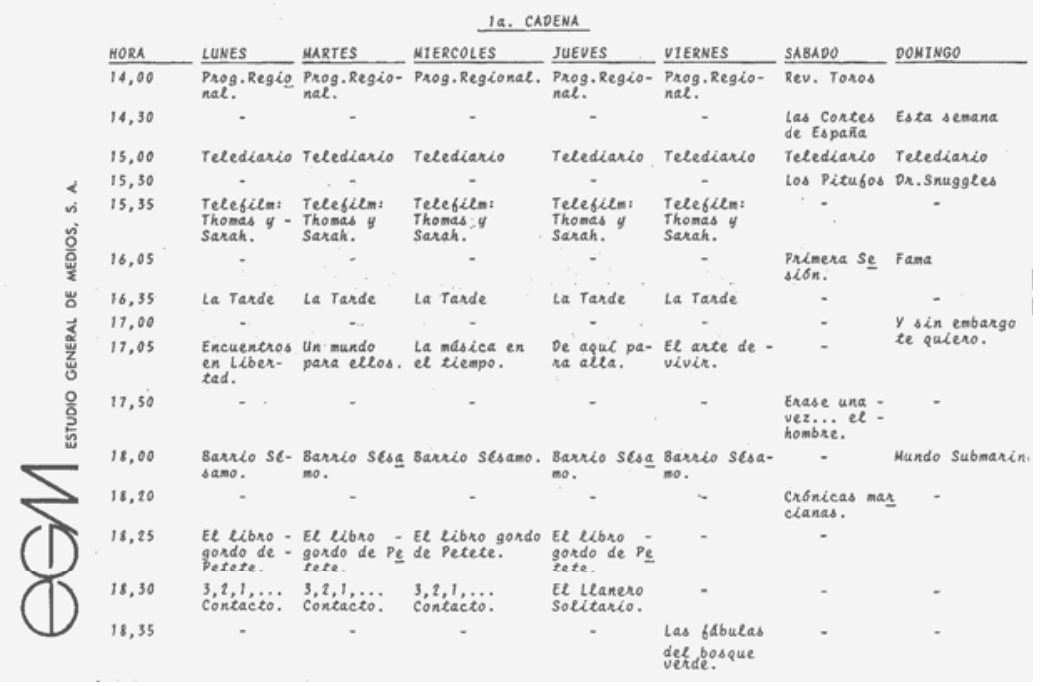




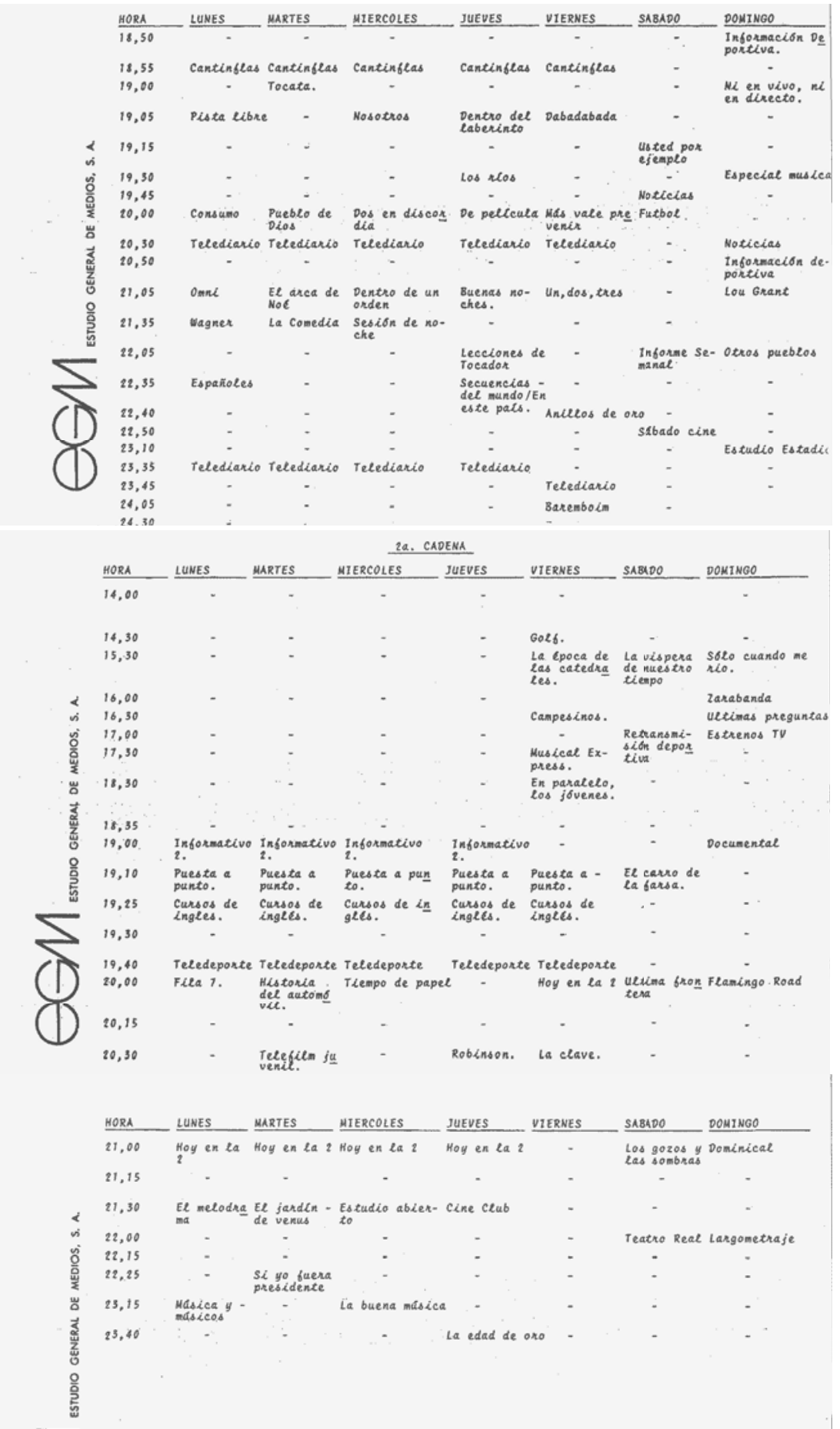


Esquema de programación de TVE para el periodo de estudio de 1990 (AIMC 1988a).

DEL 17 DE SEPTIDABRE AL 31 DE DICIDABRE DE 1990

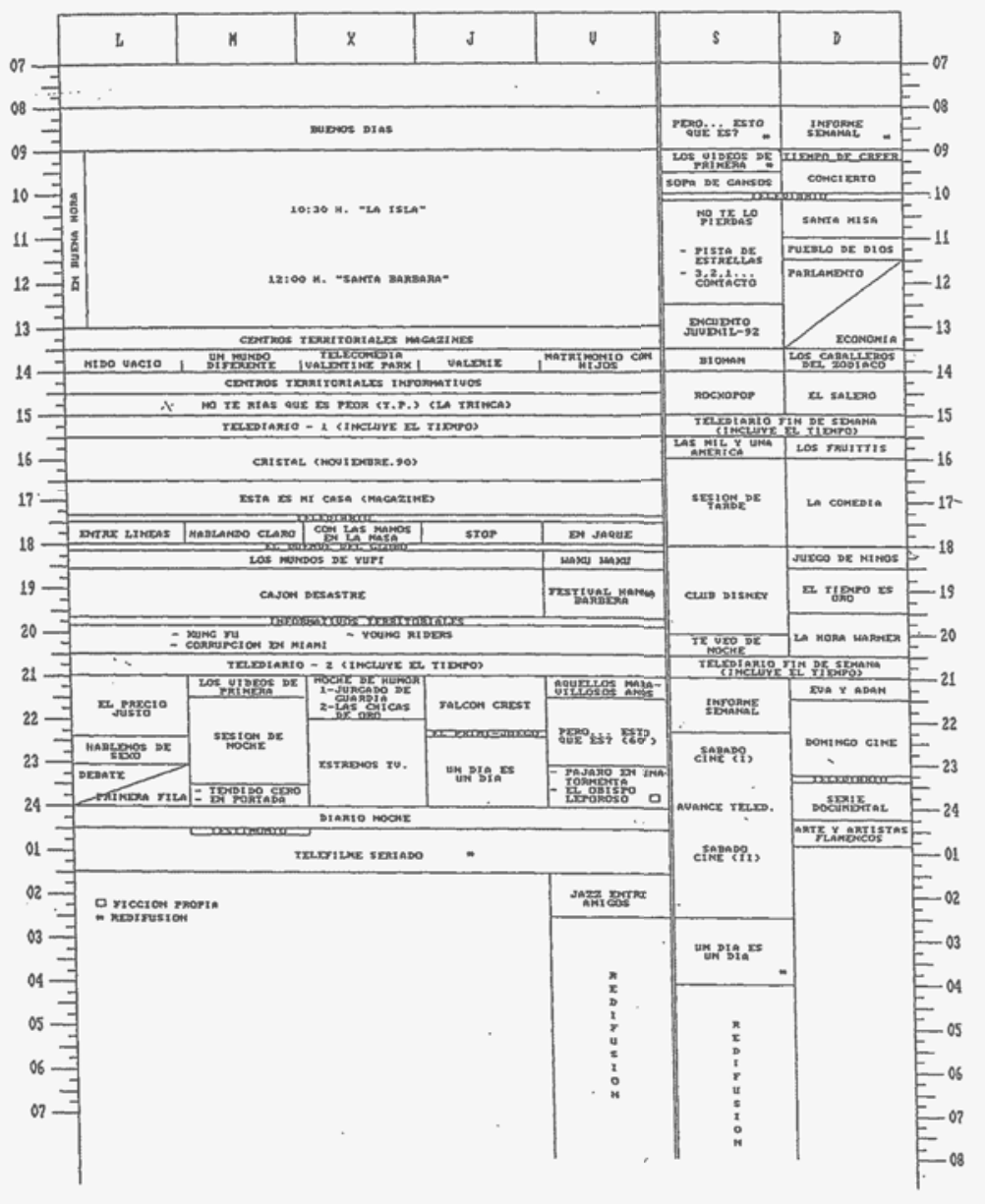


DEL 17 DE SERTIDKBRE AL 31 DE DICIDABRE DE 1990

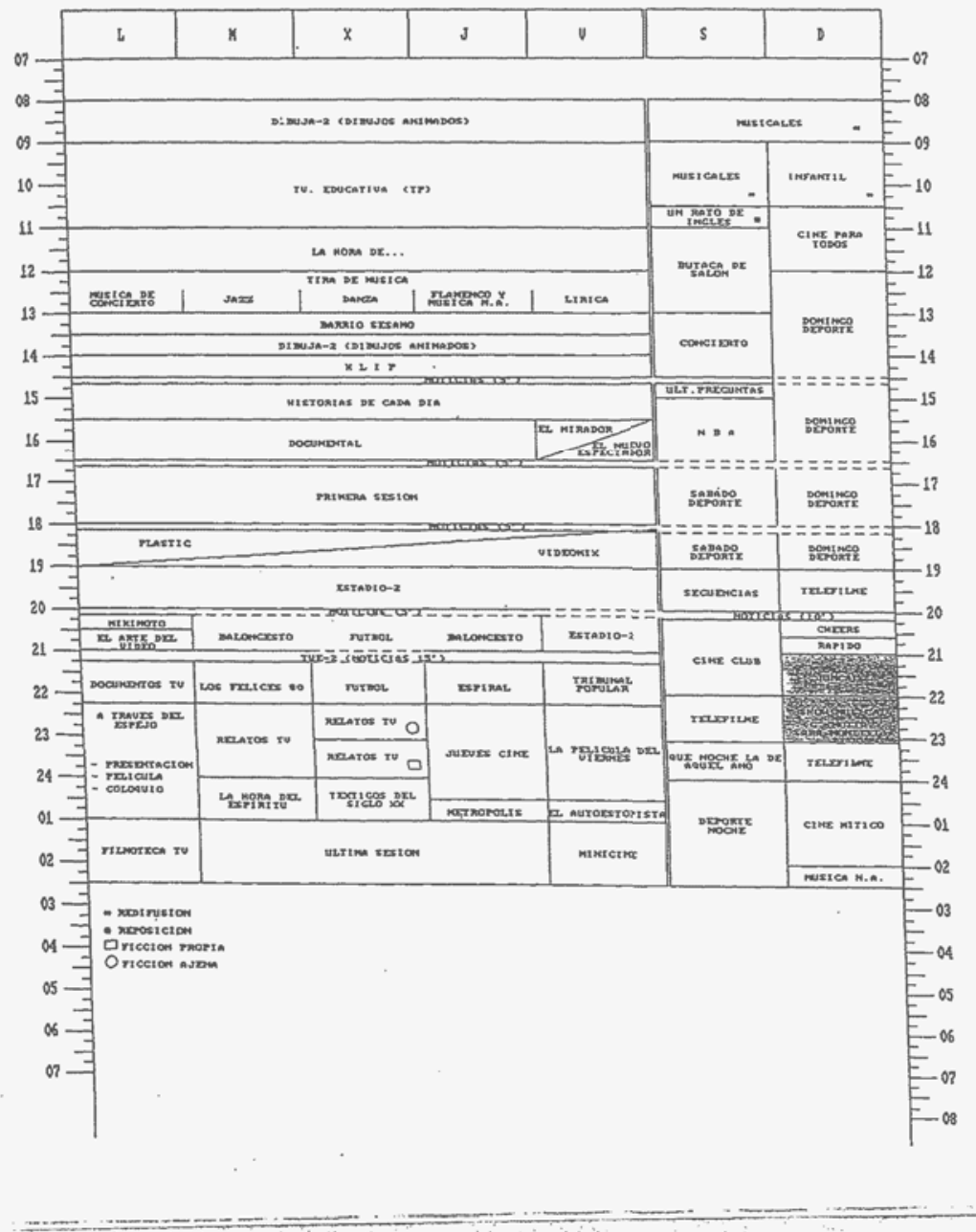

